ISSN 0103-9954

\title{
CRESCIMENTO DE CANELA-LAGEANA, Ocotea pulchella Nees et Mart. ex Nees, NA DEPRESSÃO CENTRAL DO ESTADO DO RIO GRANDE DO SUL
}

\section{GROWTH OF CANELA-LAGEANA, Ocotea pulchella Nees et Mart. ex Nees, IN THE CENTER DEPRESSION OF RIO GRANDE DO SUL STATE.}

\author{
Luciano Weber Scheeren ${ }^{1}$ Frederico Dimas Fleig ${ }^{2}$ \\ Paulo Renato Schneider ${ }^{3}$ César Augusto Guimarães Finger ${ }^{4}$
}

\section{RESUMO}

Este trabalho teve como objetivo o estudo do crescimento em diâmetro (DAP), volume comercial $(\mathrm{Vc})$, incremento corrente anual em percentagem do volume comercial (ICA\%) e a determinação do fator de forma comercial (f) para canela-lageana Ocotea pulchella Nees et Mart. ex Nees, na região da Depressão Central do estado do Rio Grande do Sul. Para o ajuste dos dados de crescimento em diâmetro e volume comercial sem casca foram testadas três equações matemáticas, sendo que a equação Quadrática resultou como modelo mais eficiente, para estimar o DAP e o Vc, ajustada em função da idade das árvores. A equação de Backman foi selecionada para o ajuste do ICA\% e do f, sendo os valores determinados em função do diâmetro à altura do peito para facilitar a aplicação prática. $\mathrm{O}$ fator de forma comercial variou desde 0,40 para um DAP de $5 \mathrm{~cm}$ até um valor máximo de 0,85 para DAP de 22 a $32 \mathrm{~cm}$, decrescendo até 0,78 aos $51 \mathrm{~cm}$ de DAP, entre as idades de 10 e 100 anos, respectivamente. $\mathrm{O}$ incremento corrente anual em percentagem do volume comercial variou de 17,48\% aos 10 anos a 1,33\% aos 100 anos de idade. A espécie apresentou um bom potencial de crescimento, o que associado com práticas de manejo poderá elevar sua produtividade.

Palavras-chave: dendrocronologia, crescimento, Ocotea pulchella.

\begin{abstract}
This work had as objectives the study of the growth in $\mathrm{DBH}$, commercial volume (CV), current annual increment in percentage of the commercial volume (CAI) and the determination of the commercial form factor (f) for Ocotea pulchella Nees et Mart. ex Nees, in the Central Depression of the Rio Grande do Sul State. The growth data were tested with three mathematical equations, for the adjustment of diameter and commercial volume without bark. The quadratic equation resulted as a more efficient model, for the CV and DBH estimate, adjusted in function of the age of the trees. The Backman equation was selected for the $\mathrm{CAI}$ and for the $\mathrm{f}$ estimate. The values were determined in function of the $\mathrm{DBH}$ to facilitate practical application. The commercial form factor varied from 0,40 for a $\mathrm{DBH}$ of $5 \mathrm{~cm}$ to a maximum value of 0,85 for a $\mathrm{DBH}$ from 22 to $32 \mathrm{~cm}$, decreasing up to 0,78 at $51 \mathrm{~cm}$ of $\mathrm{DBH}$, between the ages of 10 and 100 years. The average annual increment in percentage of the commercial volume varied from $17,48 \%$ for 10 years to $1,33 \%$ for 100 years of age. The species presented a good growth potential, that associated with management practices can increase its productivity.
\end{abstract}

Key words: dendrocronology, growth, Ocotea pulchella.

\section{INTRODUÇÃO}

Até o momento, a silvicultura brasileira teve sua atividade voltada principalmente à produção de matérias-primas para as indústrias vinculadas na transformação de madeira e fibras, tendo as plantações

1. Engenheiro Florestal, M.Sc., Doutorando pelo Programa de Pós-graduação em Engenharia Florestal, Centro de Ciências Rurais, Universidade Federal de Santa Maria, CEP 97105-900, Santa Maria (RS). a9970027@alunop.ufsm.br

2. Engenheiro Florestal, Dr., Professor do Departamento de Fitotecnia, Centro de Ciências Agroveterinárias, Universidade do Estado de Santa Catarina, CEP 88520-000, Lages (SC). a2fdf@ cav.udesc.br

3. Engenheiro Florestal, Dr., Professor Titular do Departamento de Ciências Florestais, Centro de Ciências Rurais, Universidade Federal de Santa Maria, CEP 97105-900, Santa Maria (RS). paulors@ @cr.ufsm.br

4. Engenheiro Florestal, Dr., Professor Adjunto do Departamento de Ciências Florestais, Centro de Ciências Rurais, Universidade Federal de Santa Maria, CEP 97105-900, Santa Maria (RS). finger@ccr.ufsm.br

Recebido para publicação em 4/04/2002 e aceito em 7/06/2002. 
restringido-se às espécies exóticas, por terem atendido melhor as necessidades industriais em termos de produtividade. Recentemente têm-se dado atenção ao plantio de espécies folhosas nativas, existindo poucas informações da auto-ecologia e do processo silvicultural dessas espécies para a formação de povoamentos.

Assim sendo, as plantações mistas de espécies nativas constituem o ponto central que deve ser enfocado pela silvicultura, sobretudo no sentido de obter os diferentes grupos de espécies numa plantação, adequando os espaços às exigências de cada uma delas nos diferentes períodos de formação da floresta. É nesse aspecto que a discussão de plantios das espécies arbóreas nativas deve avançar, especialmente no consórcio com outras espécies em plantios homogêneos e em floresta natural, bem como na obtenção de povoamentos puros. Nesse sentido, cabe ao manejo florestal a determinação das variáveis dendrométricas que caracterizam o crescimento das espécies nativas e fornecem parâmetros que tornem possível predizer a produção de tais povoamentos.

A Ocotea pulchella, da família das lauráceas, popularmente conhecida como canela-lageana, é uma árvore que alcança poucos metros de altura nas formações campestres, mas atinge 25-30 m de altura por 50$80 \mathrm{~cm}$ de diâmetro na floresta pluvial (Rizzini, 1971). Segundo esse mesmo autor, a madeira é empregada para tabuado, moirões e vigas, podendo ser utilizada na construção civil, sendo considerada madeira de segunda classe.

Essa espécie apresenta os limites dos anéis de crescimento, facilmente, distinguíveis. O lenho tardio possui uma coloração pardo-escura, contrastando com o lenho inicial, de coloração creme-amarelado. Do ponto de vista anatômico, observa-se macroscopicamente com o auxílio de lupa binocular (40 X), a presença de poros difusos com dimensões e distribuição uniformes em todo o lenho inicial. No lenho tardio, verificase uma diminuição no diâmetro e número de vasos, com uma maior concentração de fibras. A madeira apresenta cerne e alburno indistintos, com superfície levemente áspera. A madeira é considerada moderadamente pesada $\left(0,65 \mathrm{~g} / \mathrm{cm}^{3}\right.$ a $12 \%$ de umidade), com cheiro e gosto indistintos.

No estado do Rio Grande do Sul, a espécie é observada com frequiência nas submatas dos pinhais e no Escudo Rio-Grandense. Além disso, é encontrada nas florestas das planícies quaternárias úmidas do litoral, e com ocorrência rara nas florestas da bacia do Alto Ibicuí, Alto Uruguai e da Fralda da Serra Geral. (Reitz et al., 1988).

A carência de informações com respeito às condições de crescimento de árvores nativas existe em conseqüência da dificuldade de observações do crescimento por longos períodos. Entretanto, se uma espécie florestal apresentar anéis anuais de crescimento facilmente observáveis, a análise de tronco permite recompor o crescimento passado de maneira relativamente rápida. Em estudos dendroecológicos e dendrocronológicos, os anéis anuais em árvores mostram possíveis relações clima-crescimento e a ocorrência de perturbações passadas, antrópicas ou não.

Este trabalho tem como objetivo estudar o crescimento da canela-lageana, buscando identificar principalmente o seu crescimento em diâmetro, volume comercial, incremento corrente anual em percentagem do volume comercial sem casca e a evolução do fator de forma.

\section{MATERIAL E MÉTODOS}

As árvores de Ocotea pulchella utilizadas neste estudo foram obtidas no Campo de Instrução do Ministério do Exército (CISME), localizado no município de Santa Maria, Rio Grande do Sul. A área do estudo caracteriza-se por ser uma área de floresta natural que não sofreu qualquer intervenção nos últimos 40 anos, após a passagem de sua posse para o Ministério do Exército.

O solo é formado pela Associação Ciríaco-Charrua, sendo medianamente profundo, entre 80 a 120 $\mathrm{cm}$, moderadamente drenados, de coloração bruno-avermelhada escura, textura argilosa e desenvolvidos partindo de basaltos. Esse solo apresenta horizontes A e B bem-desenvolvidos, ligeiramente ácidos, com saturação de bases e com alumínio trocável praticamente nulo. $\mathrm{O} \mathrm{pH}$ é variável de acordo com a profundidade, passando de 6,2 no horizonte A e 5,2 no B (Lemos et al., 1973).

O clima da região, segundo a classificação de Köppen, é do tipo "Cfa", subtropical úmido, tendo como características climáticas principais a temperatura média anual de $19{ }^{\circ} \mathrm{C}$ e precipitação média anual de

Ciência Florestal, v. 13, n. 1, 2003 
1769 mm (Moreno, 1961).

A vegetação primitiva dessa região é caracterizada por uma floresta estacional decidual com ocorrência natural de canela-lageana (Reitz et al.,1983).

Neste estudo, foram utilizadas duas árvores para representar o crescimento em diâmetro, volume comercial sem casca, incremento corrente anual percentual em volume comercial e o fator de forma comercial. Foram selecionadas árvores em locais com características edáficas semelhantes e pertencentes ao estrato médio e dominante, com diâmetro à altura do peito de $51 \mathrm{~cm}$ e $38 \mathrm{~cm}$, altura comercial de $5,8 \mathrm{~m}$ e 4,5 m, e idades de 102 anos e 68 anos respectivamente. Para a determinação da idade bem como dos incrementos anuais, foi utilizada a técnica de dendrocronologia, desenvolvida por Andrew Douglass em 1920, aplicada sob fatias tomadas na base, no DAP e outras fatias tomadas até o ponto de altura comercial das árvores. Também foram tomadas fatias entre o DAP e o ponto de altura comercial das árvores, em número e posição variável no fuste para cada árvore, por causa da ocorrência de galhos ou irregularidades no tronco. Essas fatias foram etiquetadas, onde constava o número da árvore amostra e a posição de retirada da fatia no tronco. Posteriormente foram transportadas em sacos para o local de secagem.

A medição dos anéis de crescimento foi feita com o auxílio do aparelho LINTAB II, sobre dois raios marcados em cada fatia, sendo o primeiro raio marcado com um ângulo de 45 graus no sentido antihorário em relação ao maior raio da seção e o segundo raio como o prolongamento do primeiro no sentido medula-casca.

O volume comercial sem casca foi determinado pelo do método de Smalian, para seções tomadas até a altura comercial e o fator de forma comercial pela razão entre o volume comercial rigoroso pelo volume comercial cilíndrico.

Para estudar as tendências de desenvolvimento do crescimento em diâmetro e volume comercial sem casca em função da idade foram testados três modelos de regressão, apresentados na Tabela 1. Essas equações também foram ajustadas para o incremento corrente anual em percentagem do volume comercial e o fator de forma comercial em função do diâmetro à altura do peito.

TABELA 1: Equações testadas para estimar o crescimento em diâmetro, volume comercial, incremento corrente anual em percentagem do volume comercial sem casca (ICA\%) e fator de forma comercial em função da idade.

TABLE 1: Tested equations to estimate the diameter growth, commercial volume, current annual increment in percentage of the commercial volume (CAI) and commercial form factor as a function of the age.

\begin{tabular}{|c|c|c|}
\hline Equação & Modelo Matemático & Autor \\
\hline 1 & $Y=b 0 * e^{b 1 * t} t^{b 3}$ & Mitscherlich e Sonntag (1982) \\
\hline 2 & $Y=e^{b 0+b 1^{*} \ln t+b 2 * \ln 2 t}$ & Backman (1943) \\
\hline 3 & $Y=b 0+b 1{ }^{*} t+b 2 * t^{2}$ & Quadrática \\
\hline
\end{tabular}

Em que: $\mathrm{Y}$ = variável dependente, representada pelo diâmetro e volume comercial sem casca; $\mathrm{t}=$ variável independente, representada pela idade em anos; $\ln =$ logaritmo natural; b0, b1, b2 = coeficientes (Fonte: Schneider, 1993).

O processamento das equações foi realizado no pacote estatístico SPSS (Statistical Package for the Social Sciences), em computador pessoal.

\section{RESULTADOS E DISCUSSÕES}

Os resultados do processamento das equações testadas para ajustar o crescimento em diâmetro e volume comercial sem casca em função da idade, são apresentados na Tabela 2.

Observa-se que todas as equações testadas apresentaram um bom ajuste e poderiam ser utilizadas, destacando-se sobretudo as equações 1 e 3. Entretanto, considerando-se que a equação 3 (Mitserlich e Sonntag) é não-linear, a equação com utilização mais prática é a Quadrática, expressa pelo modelo 3 da Tabela 1. 
TABELA 2: Parâmetros estatísticos das equações testadas para estimar o crescimento em diâmetro e volume comercial em função da idade.

TABLE 2: Statistical parameters of the tested equations to estimate the growth in diameter and commercial volume as a function of the age.

\begin{tabular}{ll|rrr|r|r|r}
\hline \multirow{2}{*}{ Equação } & Variável Dependente & \multicolumn{3}{|c|}{ Coeficientes } & \multirow{2}{*}{$\mathrm{R}^{2}$} & $\mathrm{~S}_{\mathrm{yx}}$ & $\mathrm{CV}$ \\
\cline { 3 - 6 } & & $\mathrm{b}_{0}$ & \multicolumn{1}{c}{$\mathrm{b}_{1}$} & \multicolumn{1}{c}{$\mathrm{b}_{2}$} & & & \\
\hline 1 & Volume comercial & 0,00073 & $-1,61581$ & 1,9099200 & 0,95 & 0,06 & 16,07 \\
& Diâmetro & 0,00199 & 1,20756 & 0,9443390 & 0,84 & 0,05 & 20,26 \\
\hline 2 & Volume comercial & $-1,44400$ & $-2,88100$ & $-0,4820000$ & 0,76 & 0,08 & 23,28 \\
& Diâmetro & $-7,58200$ & 2,15100 & $-0,1410000$ & 0,74 & 0,06 & 20,55 \\
\hline 3 & Volume comercial & 0,01170 & 0,00010 & 0,0000945 & 0,95 & 0,06 & 16,07 \\
& Diâmetro & $-0,01050$ & 0,00610 & $-0,0000091$ & 0,84 & 0,05 & 20,20 \\
\hline
\end{tabular}

Em que: $\mathrm{R}^{2}=$ coeficiente de determinação; Syx = erro padrão da estimativa; $\mathrm{CV}=$ coeficiente de variação, em \%; b0, b1, b2 = coeficientes da equação.

A equação Quadrática apresentou um excelente ajuste, tanto para o diâmetro como para o volume comercial sem casca, com um coeficiente de determinação entre 0,84 e 0,95, um erro-padrão da estimativa de 0,06 para o volume comercial sem casca e 0,05 para o diâmetro, apresentando coeficiente de variação de 16,05 e 20,20\% para o volume comercial sem casca e diâmetro respectivamente.

Nas Figuras 1 e 2, encontram-se representadas as tendências de crescimento em diâmetro e volume comercial sem casca em função da idade, obtidas pela função Quadrática, expressa pelo modelo 3. Observase que, tanto para o diâmetro como para o volume comercial sem casca, o crescimento apresenta uma curva ascendente, representando que, até o momento, as árvores analisadas não atingiram a assíntota.

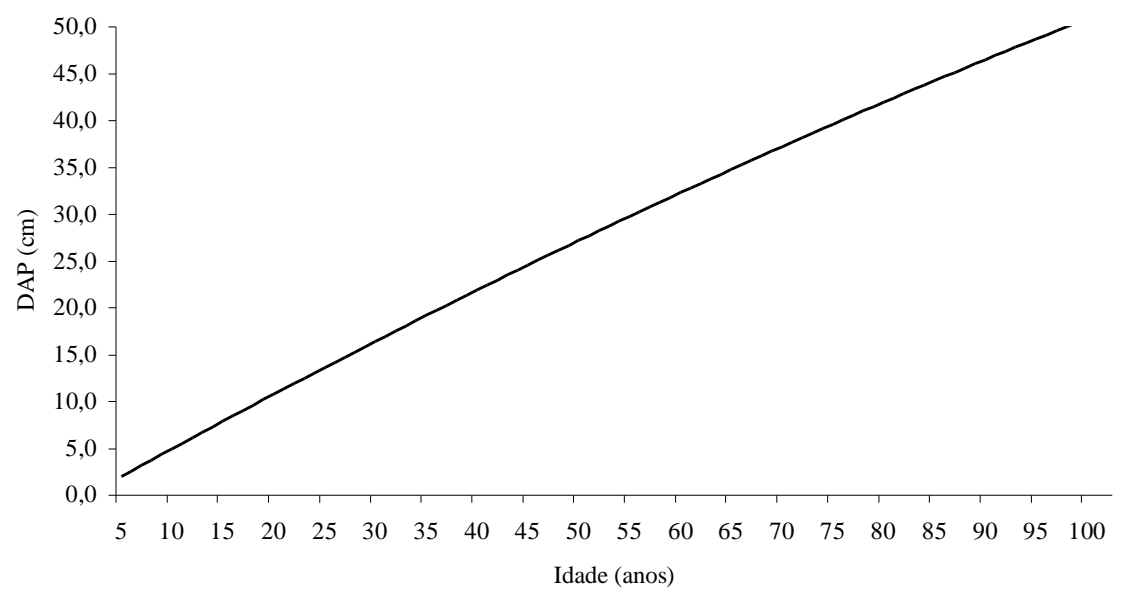

FIGURA 1: Crescimento do diâmetro à altura do peito em função da idade estimado pela equação quadrática.

FIGURE 1: DBH growth as a function of the age estimated by the quadratic equation.

Na Figura 2, observa-se que a tendência de crescimento do volume comercial sem casca em função da idade, apresenta uma forma de parábola, pois há um acréscimo constante com o tempo. Essas estimativas foram realizadas a partir dos 20 anos de idade, porque esta corresponde à idade em que as árvores atingiram a altura comercial, verificada pela presença do anel de crescimento ao nível do DAP e na altura comercial.

Com a finalidade de uso prático, as três equações foram ajustadas também para incremento corrente anual percentual em volume comercial sem casca e fator de forma comercial, como uma função do diâmetro à altura do peito, conforme é apresentado na Tabela 3. Com isso, é possível obter estimativas do incremento corrente anual em percentagem do volume comercial e do fator de forma pela medição do diâmetro à altura 
do peito que é uma variável de fácil obtenção na floresta e com baixo custo.

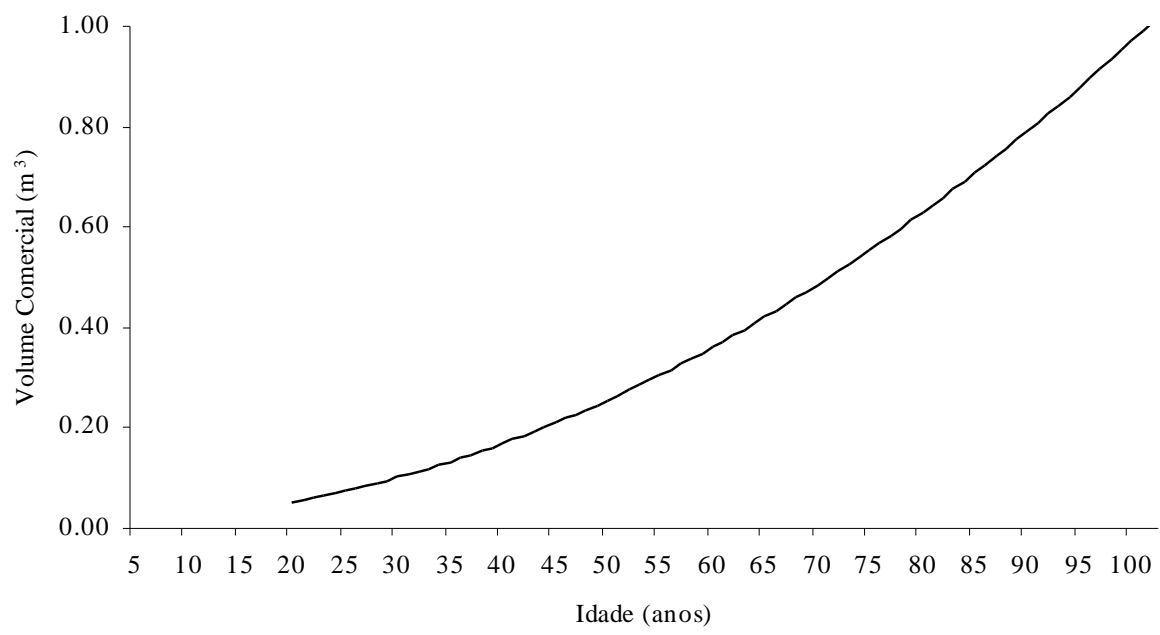

FIGURA 2: Crescimento do volume comercial sem casca em função da idade, estimado pela equação quadrática.

FIGURE 2: Commercial volume growth as a function of the age, estimated by the quadratic equation.

Para o incremento corrente anual percentual em volume comercial sem casca, a equação 2 (Backman) apresentou melhores resultados, com um coeficiente de determinação de 0,76, erro-padrão de estimativa de 0,39 e coeficiente de variação de 7,14 \%. Dessa forma, a equação de Backman foi utilizada para representar o incremento corrente anual percentual por apresentar melhor precisão estatística.

TABELA 3: Parâmetros estatísticos das equações testadas para estimar incremento corrente anual em percentagem do volume comercial sem casca e fator de forma comercial, em função do DAP.

TABLE 3: Statistical parameters of tested equations to estimate the current annual increment in percentage of the commercial volume and the commercial form factor, as a function of $\mathrm{DBH}$.

\begin{tabular}{l|l|r|r|r|r|r|r}
\hline \multirow{2}{*}{ Equação } & Variável & \multicolumn{3}{|c|}{ Coeficientes } & \multirow{2}{*}{$\mathrm{R}^{2}$} & \multirow{2}{*}{$\mathrm{S}_{\mathrm{yx}}$} & \multirow{2}{*}{ CV\% } \\
\cline { 3 - 6 } & Dependente & \multicolumn{1}{c}{$\mathrm{b}_{0}$} & $\mathrm{~b}_{1}$ & $\mathrm{~b}_{2}$ & & \\
\hline \multirow{2}{*}{1} & ICA \% & 1,369 & $-0,126$ & $-1,080$ & 0,57 & 3,44 & 60,39 \\
& Fator de forma & 0,266 & 1,231 & 0,092 & 0,19 & 0,07 & 9,24 \\
\hline \multirow{2}{*}{2} & ICA \% & $-1,444$ & $-2,881$ & $-0,482$ & 0,76 & 0,39 & 7,14 \\
& Fator de forma & $-0,581$ & $-0,655$ & $-0,254$ & 0,59 & 0,07 & 8,63 \\
\hline \multirow{2}{*}{3} & ICA \% & 22,947 & $-94,388$ & 104,758 & 0,63 & 3,22 & 59,36 \\
& Fator de forma & 0,522 & 2,043 & $-3,122$ & 0,37 & 0,07 & 8,23 \\
\hline
\end{tabular}

Em que: $\mathrm{R}^{2}=$ coeficiente de determinação; Syx = erro padrão da estimativa; $\mathrm{CV} \%$ = coeficiente de variação em \%; ICA\% = incremento corrente anual em percentagem do volume comercial sem casca; b0, b1, b2 = coeficientes da equação.

Na Figura 3, pode ser observada a tendência de desenvolvimento do incremento corrente anual percentual em volume comercial sem casca, em função do diâmetro à altura do peito, ajustada pela equação de Backman. Observa-se que o incremento corrente anual percentual em volume comercial sem casca apresentou forma exponencial negativa, diminuindo progressivamente com o aumento do diâmetro, passando de um valor máximo de $17,48 \%$, na idade de 10 anos, com um diâmetro de aproximadamente 5 cm, para um mínimo de 1,33 \%, aos 100 anos, com um diâmetro de 50,9 cm.

Os baixos valores observados podem ser atribuídos à elevada concorrência característica da floresta natural, como também às próprias características da espécie e da idade. Dessa forma, com a introdução de práticas de manejo adequado é possível elevar essas taxas de crescimento anuais, aumentando, com isso, a viabilidade econômica da espécie. 


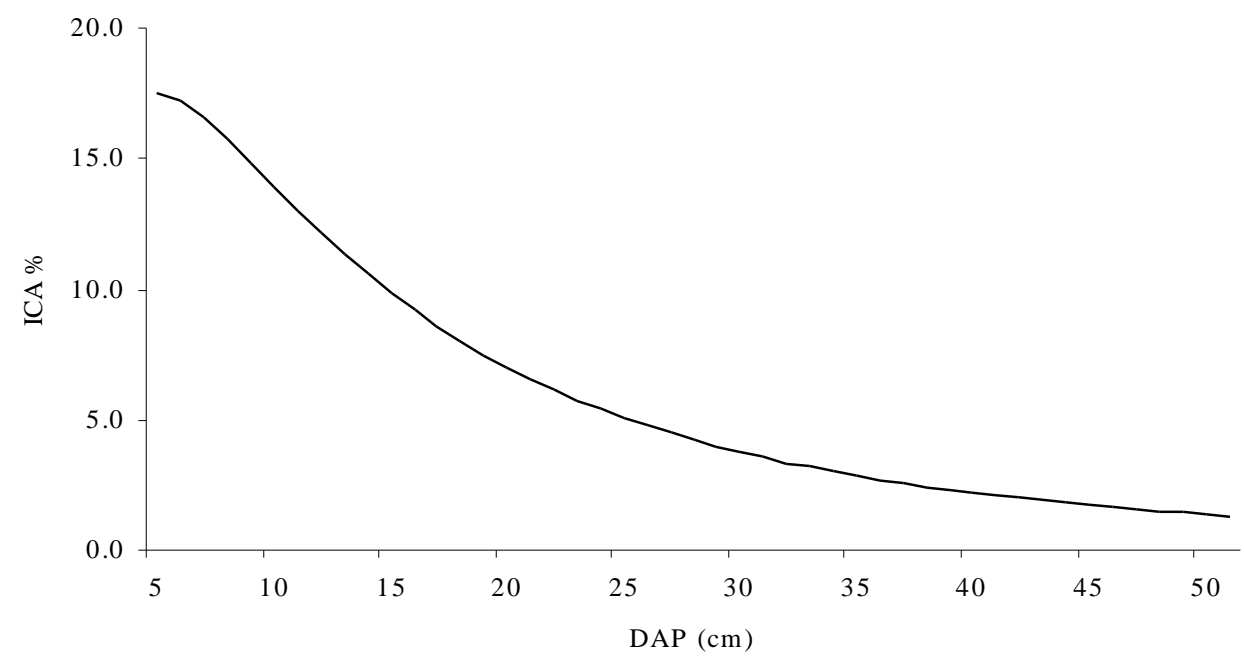

FIGURA 3: Incremento corrente anual em percentagem do volume comercial sem casca em função do DAP, estimado pela equação de Backman.

FIGURE 3: Current annual increment in percentage of the commercial volume as a function of the DBH, estimated by the Backman equation.

A equação de Backman e a equação Quadrática apresentaram precisão semelhante para o ajuste do fator de forma em função do DAP, entretanto, por causa da ligeira superioridade do coeficiente de determinação da equação de Backman, esta foi selecionada para realizar as estimativas. $\mathrm{O}$ coeficiente de determinação foi igual a 0,59, erro-padrão da estimativa de 0,07 e coeficiente de variação de 8,63 \%. Essa tendência encontra-se representada na Figura 4.

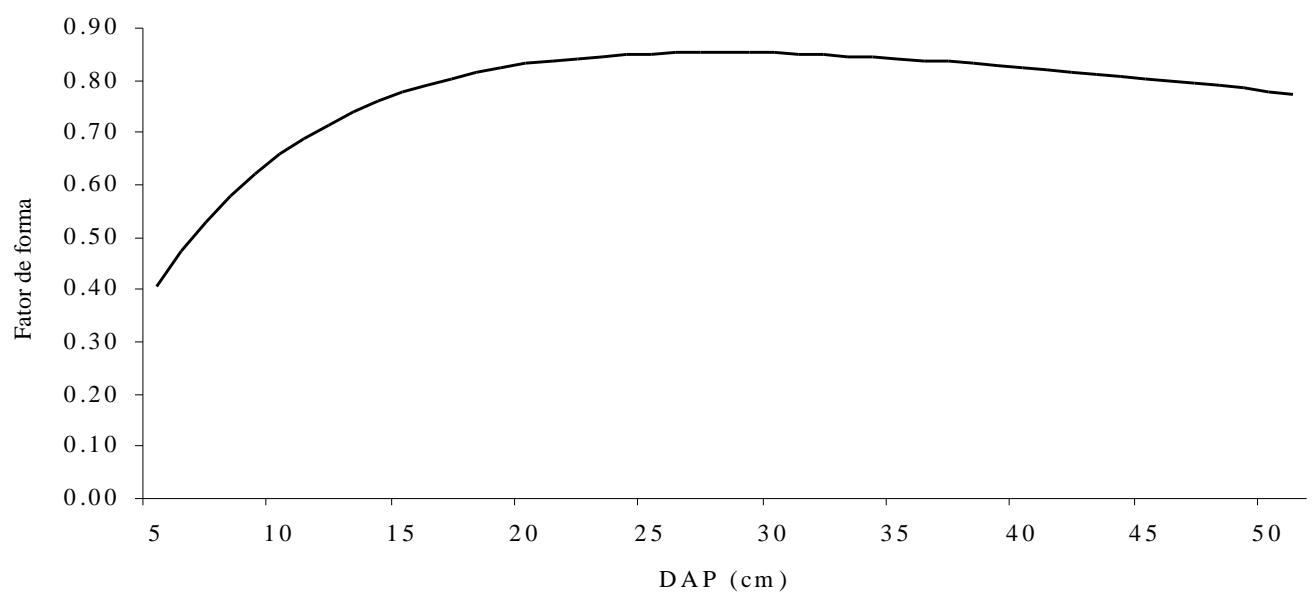

FIGURA 4: Fator de forma comercial em função do DAP, estimado pela equação de Backman.

FIGURE 4: Commercial form factor as a function of DBH, estimated by the Backman equation.

Observa-se, na Figura 4, que o fator de forma comercial apresenta tendência de crescimento com o aumento do diâmetro até atingir aproximadamente $32 \mathrm{~cm}$. O fator de forma comercial variou entre 0,40 aos $5 \mathrm{~cm}$ de diâmetro, aumentando até 0,85 entre os diâmetros de $22 \mathrm{~cm}$ a $32 \mathrm{~cm}$, e após reduzindo para um valor de 0,78 até o diâmetro máximo observado de $51 \mathrm{~cm}$. Esta variação do fator de forma comercial pode ser explicada pela dinâmica de crescimento das árvores e a sua conseqüente mudança de posição sociológica no povoamento.

$\mathrm{Na}$ Tabela 4, encontram-se os valores estimados, por idade, utilizando-se as equações selecionadas para o diâmetro à altura do peito, volume comercial sem casca, incremento corrente anual em percentagem do volume comercial sem casca e fator de forma comercial.

Ciência Florestal, v. 13, n. 1, 2003 
TABELA 4: Estimativas do crescimento do diâmetro, volume comercial sem casca, incremento corrente anual percentual em volume comercial sem casca e fator de forma comercial.

TABLE 4: Estimates of the diameter growth, commercial volume, current annual increment in percentage of the commercial volume and commercial form factor.

\begin{tabular}{c|c|c|c|c}
\hline Idade (anos) & DAP $(\mathrm{cm})$ & Volume Comercial s/c $\left(\mathrm{m}^{3}\right)$ & $\mathrm{ICA}(\%)$ & $\mathrm{f}$ \\
\hline 10 & 5,0 & 0,0226 & 17,48 & 0,40 \\
15 & 7,9 & 0,0351 & 15,86 & 0,57 \\
20 & 10,8 & 0,0524 & 13,22 & 0,68 \\
25 & 13,6 & 0,0744 & 10,84 & 0,75 \\
30 & 16,4 & 0,1011 & 8,91 & 0,80 \\
35 & 19,2 & 0,1325 & 7,38 & 0,83 \\
40 & 21,9 & 0,1687 & 6,17 & 0,84 \\
45 & 24,6 & 0,2096 & 5,21 & 0,85 \\
50 & 27,2 & 0,2552 & 4,44 & 0,85 \\
60 & 32,3 & 0,3606 & 3,31 & 0,85 \\
70 & 37,2 & 0,4849 & 2,54 & 0,83 \\
80 & 41,9 & 0,6281 & 2,00 & 0,82 \\
85 & 44,2 & 0,7068 & 1,79 & 0,81 \\
90 & 46,5 & 0,7902 & 1,61 & 0,80 \\
95 & 48,7 & 0,8783 & 1,46 & 0,79 \\
100 & 50,9 & 0,9712 & 1,33 & 0,78 \\
\hline
\end{tabular}

Em que: DAP = diâmetro à altura do peito; ICA\% = incremento corrente anual percentual em volume comercial sem casca; $\mathrm{f}=$ fator de forma comercial.

\section{CONCLUSÕES}

Os resultados obtidos nesse trabalho permitem concluir para a canela-lageana que:

a) As funções de Mitscherlich e Sonntag, Backman e Quadrática apresentaram um bom ajuste e precisão estatística para calcular as estimativas das variáveis em estudo. Por isso, qualquer uma dessas equações pode ser utilizada para realizar ajustes para a espécie em estudo.

b) $\mathrm{O}$ incremento corrente anual em percentagem do volume comercial sem casca apresentou uma tendência exponencial negativa, partindo de um valor máximo de 17,48 \%, aos 10 anos, para chegar a um valor mínimo de 1,33\%, aos 100 anos de idade. Esses valores foram observados em uma situação extrema de concorrência, demonstrando que a espécie possui um bom potencial de crescimento.

c) A própria dinâmica natural de crescimento, com sucessivas mudanças de posição sociológica das árvores dessa espécie, ocasiona um comportamento característico do fator de forma comercial que inicialmente apresentou uma tendência de aumento, atingindo um ponto de máximo que se manteve constante por um período de aproximadamente 20 anos, vindo a assumir tendência de redução até o final do período observado de 100 anos. Ficou claro que as árvores estudadas apresentaram boa forma de tronco em todas as fases de seu desenvolvimento, o que indica um ótimo aproveitamento industrial das toras.

\section{REFERÊNCIAS BIBLIOGRÁFICAS}

BACKMAN, G. Wachstum und organisches Zeit. Leipzing: Johann Ambrosis Barth, 1943. 192p.

CARVALHO, P.E. Espécies florestais brasileiras: recomedações silviculturais, potencialidades e usos da madeira. Colombo: EMBRAPA. 1994. 640p.

LEMOS, R.C.; AZOLIN, M. D.; ABRAO, P.V.R. et al. Levantamento dos solos do Estado do Rio Grande do Sul. Recife: Ministério da Agricultura - Departamento Nacional de Pesquisa Agropecuária - Divisão de Pesquisa Pedológica, 1973. 423p. (Boletim Técnico n. 301).

LORENZI, H. Árvores brasileiras: manual de identificação de plantas arbóreas nativas do Brasil. 2.ed. São Paulo: Ed. Plantarum, 1998. 368p.

MITSCHERLICH, G.; SONNTAG, G. Papelversuche: modell für eine regenerata und Neupotz-papel-ertragstafel im 
Oberheingebiet. Allg. Forst und Jg., n. 153, p. 213-219, 1982.

MORENO, J.A. Clima do Rio Grande do Sul. Porto Alegre: Secretaria da Agricultura, 1961. 41p.

PEDROSO, O.; MATTOS, J.R. Estudo sobre madeiras do Rio Grande do Sul. Porto Alegre: Instituto de Pesquisas de Recursos Naturais Renováveis "Ataliba Paz", 1987. 181p.

REITZ, R.; KLEIN, R. M.; REIS, A . Projeto madeira do Rio Grande do Sul. Porto Alegre: Secretaria de Agricultura e Abastecimento, 1983. 524p.

RIZZINI, C.T. Árvores e madeiras úteis do Brasil: manual de dendrologia brasileira. São Paulo: Edgar Blucher. Ed. USP, 1971. 244p.

SCHNEIDER, P.R. Introdução ao manejo florestal. Santa Maria: CEPEF/FATEC, 1993. 320p. 